\title{
Chemotherapeutic regimen influences the results of chemotherapy plus immunotherapy
}

\author{
Lingdi Zhao*,1 \\ ${ }^{1}$ Department of Immunotherapy, the Affiliated Cancer Hospital of Zhengzhou University \& Henan Cancer Hospital, No. 127, \\ Dongming Road, Jinshui District, Zhengzhou City 450008, China \\ *Author for correspondence: lingdi1010@126.com
}

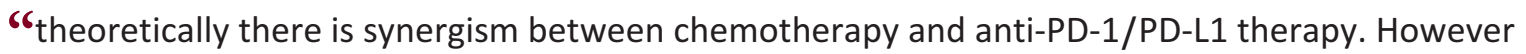
when used in the clinic, the chemotherapeutic regimen used, the doses of chemotherapeutic drugs and the sequence of chemotherapy and anti-PD-1/PD-L1 therapy could influence the efficacy of this kind of combination."

First draft submitted: 21 August 2019; Accepted for publication: 27 August 2019; Published online: 19 September 2019

The emergence of immunological checkpoint inhibitors represented by antiprogrammed death protein 1 (PD1)/PD-1 ligand (PD-L1) monoclonal antibodies (MoAb) predicts the advent of the era of cancer immunotherapy [1,2]. To date, anti-PD-1/PD-L1 antibodies are approved by US FDA in refractory/relapsed classic Hodgkin Lymphoma, melanoma, lung cancer, renal cell carcinoma, urinary tract epithelial cancer and advanced cancers with microsatellite instability-high (MSI-H), etc, and are widely used in clinics. However, the objective response rate of anti-PD-1/PD-L1 is low and a lot of patients could not benefit from anti-PD-1/PD-L1 therapy [3,4]. In order to make more patients benefit from anti-PD-1/PD-L1 MoAb therapy, anti-PD-1/PD-L1 MoAb-based combination regimens have been performed in a variety of solid tumors in clinical studies, such as anti-PD-1/PD-L1 MoAb combined with chemotherapy, radiotherapy, anticytotoxic $\mathrm{T}$ lymphocyte-associated antigen-4 MoAb, antiangiogenesis, targeted therapy, vaccine, etc [5]. The combination of anti-PD-1 MoAb and chemotherapy has been shown a synergistic effect in non-small-cell lung cancer (NSCLC) and nasopharyngeal carcinoma [6,7]. Currently, anti-PD-1 antibody combined with chemotherapy as the first-line regimen had been recommended by US-FDA in patients with advanced NSCLC without EGFR gene activating mutation and EML4-ALK fusion gene. Many clinical trials examining the clinical efficacy of anti-PD-1/PD-L1 MoAb combined with chemotherapy in solid tumors are ongoing and the results of some studies were reported on American Society of Clinical Oncology (ASCO) annual meeting this year.

The results of KEYNOTE-062 were reported at ASCO annual meeting this year. KEYNOTE-062 was a Phase III study conducted in patients with locally advanced, unresectable or metastatic gastric or gastroesophageal junction adenocarcinoma, with Her2-negative and PD-L1 combined positive score (CPS) $\geq 1$ [8]. The patients were randomly divided into three groups according to $1: 1: 1$ proportion, receiving pembrolizumab $200 \mathrm{mg} \mathrm{q} 3 \mathrm{w}$ (at most 35 cycles, $\mathrm{P}$ group), pembrolizumab $200 \mathrm{mg}$ q3w (at most 35 cycles) plus chemotherapy (P+C group) or placebo plus chemotherapy (C group). The treatments were continued until disease progression, intolerable toxicity or withdrawal from the study by researchers/patients. The primary end points were overall survival (OS) and progression-free survival. The chemotherapeutic drugs in KEYNOTE-062 were cisplatin combined with fluorouracil or capecitabine. The OS of the patients with CPS $\geq 1$ in P group, $\mathrm{P}+\mathrm{C}$ group and $\mathrm{C}$ group were 10.6, 12.5 and 11.1 months individually, while the OS of patients with CPS $\geq 10$ in the three groups were 17.5, 12.3 and 10.8 months, accordingly. Higher CPS is an important predictor for patients benefit from anti-PD-1/PD-L1 MoAb therapy; however, in patients with CPS $\geq 10$, compared with pembrolizumab monotherapy, chemotherapy combined with pembrolizumab reduced OS by 5.2 months, chemotherapy combined pembrolizumab did not play the role of one plus one over 2 , but one plus one under 1 . The possible reason underlying this may be the combined chemotherapeutic regimen, the chemotherapeutic regimen applied in this study was cisplatin plus fluorouracil or capecitabine. As application of fluorouracil requires deep venous catheterization, while capecitabine is administrated

Future $\because$ Medicine 
by oral and more convenient in clinical application, it is speculated that the majority of patients received cisplatin plus capecitabine therapy. Unfortunately, the proportion of patients received cisplatin plus capecitabine was not reported in this year ASCO annual meeting. It was reported that it took about 1 week for T-cell activation after anti-PD-1 antibody administration and activated T cells are relatively sensitive to chemotherapy, while capecitabine needs to be taken for 14 consecutive days, oral capecitabine during the 2 nd week each treatment cycle could kill activated $\mathrm{T}$ cells. This kind of regimen did not give enough time for activated $\mathrm{T}$ cells to function. This might be the main reason the $\mathrm{OS}$ of $\mathrm{P}+\mathrm{C}$ group is shorter than that of $\mathrm{P}$ group in patients with $\mathrm{CPS} \geq 10$. The chemotherapeutic regimen combined in NSCLC was pemetrexed/carboplatin and in nasopharyngeal carcinoma was gemcitabine/cisplatin [6,7]. Although gemcitabine in gemcitabine/cisplatin regimen was administrated on day 1 and 8, the half-life period of gemcitabine was only about $17 \mathrm{~min}$, the influences of gemcitabine on day 8 might be slight. And this kind of combination is conductive to exerting antitumor effect.

Cancer cell death caused by chemotherapy could result in the release of cancer-specific antigens, which are taken up by dendritic cells and antigen presenting cells nearby [9]. At the same time, chemotherapy could exert immunomodulatory effects by remodeling tumor microenvironment through reducing regulatory $\mathrm{T}$ cells and myeloid derived suppressor cells, induction of the maturation and activation of dendritic cells, enhancement effector T-cell function and upregulation of PD-L1 expression on tumor cells [10]. So theoretically there is synergism between chemotherapy and anti-PD-1/PD-L1 therapy. However when used in the clinic, the chemotherapeutic regimen used, the doses of chemotherapeutic drugs and the sequence of chemotherapy and anti-PD-1/PD-L1 therapy could influence the efficacy of this kind of combination. Although the primary end point was not achieved in KEYNOTE-062, this does not mean that chemotherapy combined with PD-1/PD-L1 regimen in patients with advanced gastric cancer could not be performed as front-line therapy. When treating patients, we should take many into consideration, such as the patients' performance status, the impact of the tumor on the patient, the biological characteristics of the tumor, the patients' treatment wish, etc. For the patients with CPS $\geq 10$ without MDM2/MDM4 amplification and the performance status Eastern Cooperative Oncology Group (KPS ECOG) $\leq 2$, pembrolizumab monotherapy might be a good choice, but if the tumor burden is huge, or existence of tumor compression or other symptoms which needs to reduce the tumor burden rapidly, pembrolizumab combined with chemotherapy might be better, but the drugs in the chemotherapeutic regimen selected should not be given more than 1 week. If there are contraindications for the patients to receive anti-PD-1/PD-L1 therapy, chemotherapy might be the good choice. For patients with CPS $\geq 1$, pembrolizumab monotherapy should be taken into consideration if there is chemotherapy contraindications, and pembrolizumab combined chemotherapy should be taken into consideration if there is no chemotherapy contraindications. As for the chemotherapeutic regimen combined, cisplatin or oxaliplatin combined with fluorouracil or irinotecan/fluorouracil in which all the chemotherapeutic drugs were administrated in 1 week might be better.

Chemotherapeutic drugs could kill the tumor cells, and at the same time kill the immunosuppressive cells in the tumor microenvironment. The immunosuppressive cells (such as regulatory $\mathrm{T}$ cells and myeloid derived suppressor cells) in tumor microenvironment are in a relatively proliferating active status and sensitive to chemotherapy, the toxicity of chemotherapeutic drugs on local immunosuppressive cells is beneficial for lymphocytes entering the tumor site and exert antitumor activity. Although more and more cancer therapists realize that chemotherapy possesses immunomodulatory effects, the dosage of different chemotherapeutic drugs that could exert optimal killing effects on immunosuppressive cells is still unclear and the optimal dosage of chemotherapy should be explored. Reducing the dose intensity of chemotherapeutic drugs might lead to a better clinical efficacy when combined with immunotherapy.

In clinical practice, we should not only acquire experience from successful clinical studies, but also from the failed clinical studies. We should confirm our practice according to the guidelines, at the same time, individualized comprehensive treatment modalities should be adopted according to the mechanism of action of the combined regimen, the interactions between the drugs combined, the patients' performance status, the biological characteristics of the tumors, etc. These will allow the patients to benefit most from the treatment selected.

Financial \& competing interests disclosure

The author has no relevant affiliations or financial involvement with any organization or entity with a financial interest in or financial conflict with the subject matter or materials discussed in the manuscript. This includes employment, consultancies, honoraria, stock ownership or options, expert testimony, grants or patents received or pending, or royalties.

No writing assistance was utilized in the production of this manuscript. 


\section{References}

1. Topalian SL, Hodi FS, Brahmer JR et al. Safety, activity, and immune correlates of anti-PD-1 antibody in cancer. N. Engl. J. Med. 28, 366(26), 2443-2454 (2012).

2. Brahmer JR, Tykodi SS, Chow LQ et al. Safety and activity of anti-PD-L1 antibody in patients with advanced cancer. N. Engl. J. Med. 28, 366(26), 2455-2465 (2012).

3. Wang M, Ma X, Guo L et al. Safety and efficacy profile of pembrolizumab in solid cancer: pooled reanalysis based on randomized controlled trials. Drug Des. Devel. Ther. 11, 2851-2860 (2017).

4. Jiang C, Cai X, Zhang $\mathrm{H}$ et al. Activity and immune correlates of a programmed death-1 blockade antibody in the treatment of refractory solid tumors. J. Cancer 9(1), 205-212 (2018).

5. Du Rusquec $\mathrm{P}$, de Calbiac O, Robert $\mathrm{M}$ et al. Clinical utility of pembrolizumab in the management of advanced solid tumors: an evidence-based review on the emerging new data. Cancer Manag. Res. 11, 4297-4312 (2019).

6. Gandhi L, Rodriguez-Abreu D, Gadgeel S et al. Pembrolizumab plus chemotherapy in metastatic non-small cell lung cancer. $N$. Engl. J. Med. 378, 2078-2092 (2018).

7. Fang W, Yang Y, Ma Y et al. Camrelizumab (SHR-1210) alone or in combination with gemcitabine plus cisplatin for masopharyngeal carcinoma: results from two single-arm, phase 1 trial. Lancet Oncol. 19(10), 1338-1350 (2018).

8. Tabernero J, Van Cutsem E, Bang YJ et al. Pembrolizumab with or without chemotherapy versus chemotherapy for advanced gastric or gastroesophageal junction (G/GEJ) adenocarcinoma: the phase III KEYNOTE-062 study. ASCO LBA(4007), (2019).

9. Chen DS, Mellman I. Oncology meets immunology: the cancer-immunity cycle. Immunity 39(1), 1-10 (2013).

10. Cruz E, Kayser V. Monoclonal antibody therapy of solid tumors: clinical limitations and novel strategies to enhance treatment efficacy. Biol. Targets Ther. 13, 33-51 (2019). 
